INTERVENTIONAL CARDIOLOGY AND SURGERY

\title{
Assessment of left ventricular function long term after arterial switch operation for transposition of the great arteries by dobutamine stress echocardiography
}

\author{
L Hui, A K T Chau, M P Leung, C S W Chiu, Y F Cheung
}

Heart 2005;91:68-72. doi: 10.1136/hrt.2003.027524

See end of article for authors' affiliations

Correspondence to: Dr Y F Cheung, Division of Paediatric Cardiology, Department of Paediatrics and Adolescent Medicine Grantham Hospital, The University of Hong Kong, 125 Wong Chuk Hang Road, Aberdeen, Hong Kong, People's Republic of China; xfcheung@hkucc. hku.hk

Accepted 24 March 2004
Objectives: To use dobutamine stress echocardiography to determine left ventricular (LV) function and wall motion of children long term after arterial switch operation (ASO) for transposition of the great arteries. Design and patients: 31 patients (24 boys) with ASO performed at a mean (SD) of 15.5 (4.3) days of life were studied at an age of 9.4 (2.0) years. All had normal coronary angiographic findings. LV echocardiographic indexes, including fractional shortening, ejection fraction, rate corrected velocity of circumferential fibre shortening (VCFc), and wall stress, as well as LV wall motion abnormalities were determined at rest and under dobutamine stress. The results were compared with those of 20 healthy age matched control participants.

Setting: Tertiary paediatric cardiac centre.

Results: Fractional shortening, ejection fraction, and VCFc were significantly lower in patients than in controls at rest (all with $p<0.001$ ). Stress-velocity index detected impaired LV contractility in 19 (61\%) patients at rest. An older age at operation $(p=0.01)$, longer bypass $(p=0.01)$ and circulatory arrest times $(p=0.045)$, and an unusual coronary artery pattern $(p=0.059)$ were associated with impaired resting LV contractility. Dobutamine stress echocardiography unmasked wall motion abnormalities in 23 (74\%) patients. Exercise myocardial perfusion scan, performed in 22 patients, showed reversible myocardial perfusion defects in 17. These defects corresponded to segments of hypokinesia as detected by dobutamine stress echocardiography.

Conclusion: A significant proportion of children, albeit asymptomatic, had impaired baseline LV contractility and reversible myocardial perfusion defects and mild wall motion abnormalities on stress after ASO.
A rterial switch operation (ASO) is the treatment of choice for transposition of the great arteries (TGA), ${ }^{12}$ with encouraging short and intermediate term follow up results being reported..$^{1-7}$ Load dependent echocardiographic indexes in previous studies have shown normal systolic and diastolic function of the left ventricle at short and intermediate term follow up. ${ }^{357}$ Further studies of the left ventricular (LV) end systolic wall stress-velocity of fibre shortening relation, a preload independent, afterload adjusted index, have also shown normal LV contractility at intermediate term follow up. ${ }^{4}$ However, as long term success of ASO depends on adequacy of the coronary arterial circulation among other factors, the need for coronary artery implantation in ASO remains a cause for concern. Recently, impaired myocardial blood flow during vasodilatation with adenosine and attenuation of coronary flow reserve have been observed in asymptomatic children with TGA a decade after ASO. ${ }^{8}$ Although the clinical consequences are unclear, these findings suggest a possibility of long term stress induced LV dysfunction in patients after ASO.

Dobutamine stress echocardiography (DSE) has been used for early detection of LV dysfunction in long term childhood cancer survivors ${ }^{9}$ and children with thalassaemia ${ }^{10}$ through evaluation of myocardial contractile reserve. Furthermore, DSE has been shown to be useful in the diagnosis of myocardial ischaemia secondary to congenital or acquired coronary artery abnormalities. ${ }^{11}{ }^{12}$ To our knowledge, DSE has not been applied to evaluate LV function in children with TGA after ASO. Given the previous findings of reduced coronary flow reserve after ASO and sensitivity of DSE in early detection of myocardial dysfunction, we hypothesised that DSE can unmask LV dysfunction in apparently asymptomatic children with TGA after ASO in the long term. To examine this hypothesis, we determined the LV function of 31 children with TGA at a mean of 9.4 years after ASO, at rest, and after intravenous dobutamine infusion, and compared the findings with those of a healthy control group. To evaluate further the significance of abnormal DSE findings, we recorded exercise myocardial perfusion scans in 22 of the 31 patients.

\section{METHODS}

\section{Patients}

Thirty one patients ( 24 boys) who had undergone ASO for TGA were studied at an age of 9.4 (2.0) years (range 7-14 years). All had simple TGA with an intact ventricular septum. ASO was performed at 15.5 (4.3) days (range 8-25 years) of life. A single surgeon (CSWC) performed all the operations. The cardiopulmonary bypass time was 130 (17) minutes, aortic cross clamp time was 90 (19) minutes, and circulatory arrest time was 6 (9) minutes. Coronary artery implantations were performed without complications and the postoperative course was uneventful in all of the patients. The usual type of coronary arterial anatomy (1LCx;2R, Leiden convention) ${ }^{13}$

Abbreviations: ASO, arterial switch operation; DSE, dobutamine stress echocardiography; EF, ejection fraction; ET, ejection time; FS, fractional shortening; LV, left ventricular; LVEDD, left ventricular end diastolic dimension; LVESD, left ventricular end systolic dimension; LVESP, left ventricular end systolic pressure; LVPWS, left ventricular posterior wall thickness at systole; TGA, transposition of the great arteries; VCFc, rate corrected velocity of circumferential fibre shortening 


\begin{tabular}{|ll|}
\hline $\begin{array}{l}\text { Table } 1 \\
\text { (Leiden convention) }\end{array}$ & Coronary artery anatomy of patients \\
\hline Coronary artery anatomy & Number \\
\hline Usual (1LCx;2R) & 25 \\
Other & 3 \\
$1 \mathrm{~L} ; 2 \mathrm{C} x \mathrm{R}$ & 2 \\
$2 \mathrm{LC} \times \mathrm{R}$ & 1 \\
$\mathrm{ILCxR}$ & \\
\hline Cx, circumflex artery; L, left; $R$, right. \\
\hline
\end{tabular}

was present in 25 patients; table 1 summarises the other types. Twenty healthy children (seven boys) aged 10.4 (4.5) years (range $4-15$ years) $(\mathrm{p}=0.34)$ were recruited as controls. The institutional ethics committee approved the study and the parents of all patients gave written informed consent.

\section{Dobutamine stress echocardiography Protocol}

Transthoracic echocardiography was performed with the Vingmed System 5 (Horten, Norway) ultrasound machine. Baseline echocardiographic recordings were made after 15 minutes of rest. Continuous dobutamine infusion was then initiated at $5 \mu \mathrm{g} / \mathrm{kg} / \mathrm{min}$ and increased stepwise to 10 and $20 \mu \mathrm{g} / \mathrm{kg} / \mathrm{min}$. Echocardiographic examination was repeated after 10 minutes of drug infusion at each stage to ensure that a steady state concentration of dobutamine was attained. Dobutamine infusion was terminated prematurely in the presence of any of the following: an increase in systolic blood pressure of $>30 \%$ above baseline or a decrease of $>20 \%$ below baseline; an increase in heart rate of $40 \%$ above baseline; cardiac arrhythmias; and significant symptoms or discomfort.' Systemic blood pressure of the right arm was measured with an automatic oscillometric device (Dinamap, Critikon Inc, Tampa, Florida, USA). At baseline and each stage of dobutamine infusion, echocardiogram, ECG, and systemic blood pressure were recorded simultaneously.

\section{Assessment of LV contractility}

The standard parasternal short axis view just below the tips of the mitral valve leaflets was used to derive the following $M$ mode measurements ${ }^{14}$ at baseline and during infusion of dobutamine: LV systolic dimension, LV end diastolic dimension (LVEDD), and LV posterior wall thickness at systole (LVPWS). Fractional shortening (FS) and ejection fraction (EF) of the left ventricle were calculated according to standard formulas: $\mathrm{FS}=($ LVEDD - LVESD $) /$ LVEDD, where LVESD is LV end systolic dimension; and $\mathrm{EF}=\left(\mathrm{LVEDD}^{3}-\mathrm{LVESD}^{3}\right) / \mathrm{LVEDD}^{3}$. The aortic ejection time (ET) and RR interval were measured by pulsed Doppler and from the surface ECG, respectively. Rate corrected velocity of circumferential fibre shortening (VCFC) was calculated by $\sqrt{ } \mathrm{RR} \times \mathrm{FS} / \mathrm{ET}$ and LV systolic meridional wall stress was calculated by $(0.337 \times$ LVESP $\times$ LVESD $) /$ LVPWS ( 1 + LVPWS/LVESD), where LVESP is LV end systolic pressure. $^{6}$ Echocardiographic assessments were analysed off line blinded to patient status. Average values of indexes obtained from three consecutive cardiac cycles were used for analysis. The peak systolic pressure was used to estimate LVESP. ${ }^{15}$ The reference LV myocardial contractility index, a relatively load independent index based on the relation between VCFC and wall stress, ${ }^{16}$ was derived from controls by linear regression. In the absence of LV outflow obstruction, the relation between VCFc and stress at peak systole correlates well with the relation between VCFc and stress at end systole. ${ }^{15}$ Patients with data points lying below two standard deviations of normal were regarded as having impaired myocardial contractility.

\section{Wall motion analysis}

Two dimensional images of the apical four chamber, apical two chamber, parasternal long axis, and parasternal short axis views at baseline and all stages of dobutamine infusion were acquired digitally for three consecutive cardiac cycles and analysed off line on a quad screen display. The LV wall was divided into 16 segments for analysis of wall motion, which was scored according to a four point scale: 1 for normokinesia, 2 for hypokinesia, 3 for akinesia, and 4 for dyskinesia. ${ }^{17}$ The overall LV wall motion score was then calculated as the sum of scores in visualised segments divided by the number of segments visualised. LV wall motion of the first 16 patients was analysed separately by one of the authors (LH) and a single adult cardiologist, and the intraclass correlation coefficient of wall motion score assessment by the two assessors was 0.96 .

\section{Exercise myocardial perfusion scintigraphy}

Of the 31 patients, 22 agreed to have an exercise myocardial perfusion scan with either thallium-201 $(\mathrm{n}=2)$ or technetium-99m tetrofosmin $(\mathrm{n}=20)$. The treadmill exercise testing was performed according to the Bruce protocol. For ${ }^{201} \mathrm{Tl}$ scintigraphy, ${ }^{201} \mathrm{Tl}$ thallous chloride was injected intravenously at peak exercise at a dose of $2 \mathrm{mCi} / 60 \mathrm{~kg}$ body weight. Cardiac emission computed tomograms were obtained at 15 minutes and four hours after exercise. For tetrofosmin scintigraphy, ${ }^{99 \mathrm{~m}} \mathrm{Tc}$ tetrofosmin was injected intravenously at peak exercise at a dose of $25 \mathrm{mCi} / 60 \mathrm{~kg}$. Gated cardiac emission computed tomograms were obtained 30 minutes after injection. On the following day, ${ }^{99 \mathrm{~m}} \mathrm{Tc}$ tetrofosmin was injected intravenously at rest at a dose of $25 \mathrm{mCi} / 60 \mathrm{~kg}$. Cardiac emission computed tomograms were obtained one hour after injection. Locations of perfusion defects, if present, were noted and compared with wall motion abnormalities as detected by DSE. The perfusion defects were described according to the spatial distribution as anterior, lateral, posterior, inferior, septal, apical, or a combination of these.

\section{Statistical analysis}

Data are expressed as mean (SD) unless otherwise stated. Echocardiographic indexes of the patient and control groups were compared by unpaired Student's $t$ test. Demographic and perioperative factors of patients with impaired LV contractility were compared with those of patients with normal contractility by unpaired Student's $t$ test or Fisher's exact test where appropriate. As segmental descriptions of wall motion and perfusion defects differed, it was impossible to calculate the $\kappa$ value for determination of agreement and comparisons were made generically. All tests were two tailed and $\mathrm{p}<0.05$ was considered significant.

\section{RESULTS \\ Patients}

The patients were studied at a mean (SD) age of $9.4(2.0)$ years (range 7-14 years) after ASO. At the time of the study, all were in New York Heart Association functional class I and none had symptoms of myocardial ischaemia. All of the patients underwent cardiac catheterisation with selective coronary arteriograms at an age of 4.6 (2.5) years. None had occlusion or stenosis of the reimplanted coronary arteries. In one patient, however, mild branch pulmonary artery stenosis was noted. There were no significant differences in body weight (31.7 (9.3) kg $v 36.2(9.5) \mathrm{kg}, \mathrm{p}=0.10)$, systolic blood pressure (105 (10) $\mathrm{mm} \mathrm{Hg} v 109$ (12) $\mathrm{mm} \mathrm{Hg}$, $\mathrm{p}=0.20$ ), diastolic blood pressure (54 (8) $\mathrm{mm} \mathrm{Hg} v 56$ 
Table 2 Comparison of baseline left ventricular echocardiographic indexes between patient and control groups

\begin{tabular}{llll}
\hline Variable & Patients $(\mathbf{n}=\mathbf{3 1})$ & Controls $(\mathbf{n}=20)$ & $\mathrm{p}$ Value \\
\hline HR (beats/min) & $81(14)$ & $78(13)$ & 0.46 \\
FS (\%) & $0.33(0.05)$ & $0.41(0.05)$ & $<0.001^{*}$ \\
EF $(\%)$ & $0.70(0.06)$ & $0.80(0.06)$ & $<0.001^{*}$ \\
VCFc (circ/s) & $0.97(0.18)$ & $1.23(0.21)$ & $<0.001^{*}$ \\
LVSD $(\mathrm{cm})$ & $2.43(0.48)$ & $2.47(0.36)$ & 0.74 \\
LVPWS $(\mathrm{cm})$ & $1.28(0.29)$ & $1.11(0.17)$ & $0.01^{*}$ \\
\hline
\end{tabular}

$E F$, ejection fraction; FS, fractional shortening; HR, heart rate; LVPWS, left ventricular posterior wall thickness at systole; LVSD, left ventricular systolic dimension; VCFc, rate corrected velocity of circumferential fibre shortening.

*Significant difference.

(7) $\mathrm{mm} \mathrm{Hg}, \mathrm{p}=0.46$ ), and resting heart rate (81 (14) beats/ min $v 78$ (13) beats/min, $\mathrm{p}=0.46$ ) between patients and controls.

\section{Baseline LV wall motion and systolic function}

None of the patients and control subjects had wall motion abnormalities at baseline. Table 2 summarises the baseline LV echocardiographic indexes. The FS, EF, and VCFC were significantly lower in patients than in controls (all with $\mathrm{p}<0.001)$. Nonetheless, only two of the 31 patients had an FS below normal, both of which measured $26 \%$. On the basis of the stress-velocity relation, 19 of $31(61 \%)$ patients had impaired LV contractility (fig 1). When compared with those having normal LV contractility $(\mathrm{n}=12)$, patients with impaired contractility $(\mathrm{n}=19)$ had significantly lower FS (32 (5)\% v 35 (4)\%, p < 0.001), EF (69 (6)\% $v 72(6) \%$, $\mathrm{p}<0.001)$, VCFC $(0.90 \quad(0.16) \mathrm{circ} / \mathrm{s} v 1.06(0.17) \mathrm{circ} / \mathrm{s}$, $\mathrm{p}<0.001)$, and wall stress $\left(43.6 \quad(19.1) \mathrm{g} / \mathrm{cm}^{2} \vee 54.4\right.$ (22.1) $\left.\mathrm{g} / \mathrm{cm}^{2}, \mathrm{p}<0.001\right)$. Factors associated with impaired LV contractility were an older age at operation $(\mathrm{p}=0.01)$ and longer cardiopulmonary bypass $(\mathrm{p}=0.01)$ and circulatory arrest times $(\mathrm{p}=0.045)$. An unusual coronary artery pattern also tended to be associated with impaired LV contractility $(\mathrm{p}=0.059)($ table 3$)$.

\section{Dobutamine stress echocardiography}

The maximum tolerated dose of dobutamine was 5, 10, and $20 \mu \mathrm{g} / \mathrm{kg} / \mathrm{min}$ in two, 27, and two patients, respectively. The cause of termination was hypertension in 23 patients, arrhythmia in seven, tachycardia in six, chest pain in three,

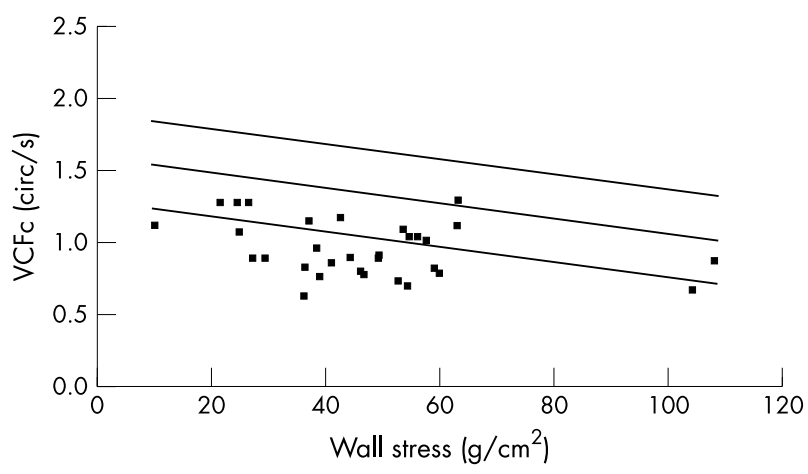

Figure 1 Baseline stress-velocity index of patients. Solid squares represent data points derived from patients after arterial switch operation. The linear regression lines represent mean (2SD) as derived from healthy age matched control subjects. Data points lying below 2SD of normal were regarded as indicating impaired myocardial contractility. VCFc, corrected velocity of circumferential fibre shortening. hypotension in one, and headache in one. Eleven patients had more than one of the above symptoms.

\section{Wall motion abnormalities and LV systolic function under dobutamine stress}

Segmental wall motion was analysed at the maximum tolerated dose of dobutamine. At the maximum tolerated dose, 22 patients had mild hypokinesia of one or more segments, and one had akinesia and dyskinesia involving the apical anterior septal, mid anterior septal, and basal anterior septal segments. All of these patients had impaired LV contractility at baseline, which included the six with unusual coronary artery anatomy. Overall, the wall motion score was $1.02(0.03)$ at rest, $1.41(0.37)$ at $5 \mu \mathrm{g} / \mathrm{kg} / \mathrm{min}$ of dobutamine infusion, $1.43(0.38)$ at the maximum tolerated dose, and 1.03 (0.06) after termination of dobutamine infusion. In the 29 patients who tolerated $>5 \mu \mathrm{g} / \mathrm{kg} / \mathrm{min}$ of dobutamine infusion, their wall motion score at $5 \mu \mathrm{g} / \mathrm{kg} / \mathrm{min}$ of dobutamine infusion did not differ significantly from that at the maximum tolerated dose of dobutamine (1.41 (0.37) $v 1.43$ (0.38), $\mathrm{p}=0.83)$. No segmental wall motion abnormalities were detected in controls, both at baseline and on dobutamine stress. Derivations of wall stress and the stress-velocity index were not attempted in light of the observed wall motion abnormalities in $74 \%$ ( 23 of 31 ) of patients.

\section{Exercise myocardial perfusion scan findings}

Of the 22 patients who had undergone exercise myocardial perfusion scan, 17 were found to have partially reversible myocardial perfusion defects. Locations of these perfusion defects corresponded to segments of hypokinesia as detected by DSE (table 4). Furthermore, all of these 17 patients had impaired contractility, as defined by the stress-velocity relation, at baseline. In the remaining five patients who had normal perfusion scan results, the baseline LV cardiac contractility was normal and the LV wall motion remained normal under stress.

\section{DISCUSSION}

This study shows that even at baseline, long term LV contractility is abnormal in a significant proportion $(61 \%$, 19 of 31) of patients with TGA after ASO. The use of DSE further unmasks wall motion abnormalities in $74 \%$ (23 of 31 ) of patients. Although none of the patients had symptoms of myocardial ischaemia or angiographic evidence of coronary artery abnormalities, reversible myocardial perfusion defects were observable in those with impaired LV contractility. Furthermore, corresponding wall motion abnormalities, albeit mild, were detected in these segments by DSE. The absence of a well defined coronary arterial territory involvement, however, suggests global, rather than regional, involvement. Older age at operation, longer cardiopulmonary bypass time, longer circulatory arrest time, and an unusual coronary artery pattern were associated with impaired LV contractility.

Previous studies have reported encouraging short and intermediate term outcomes of children with TGA after ASO. Lupinetti and colleagues ${ }^{3}$ reported that, of their 115 patients who were assessed by echocardiography at a mean duration of 12 months after ASO, all but one had normal FS and LV wall motion. ${ }^{3}$ Similarly, Hovels-Gurich and colleagues ${ }^{5}$ observed normal LV shortening fraction in 77 children assessed at a mean of 5.4 years after ASO. Colan and colleagues $^{4}$ further used a preload independent, afterload adjusted myocardial contractility index, and showed that LV contractility was normal in 32 patients at five to 50 months after ASO. These findings were replicated in their later study of 330 children with TGA at a mean of 3.5 years after ASO, 184 of whom had an intact ventricular septum. ${ }^{6}$ 
Table 3 Factors associated with impaired left ventricular contractility

\begin{tabular}{|c|c|c|c|}
\hline \multirow[b]{2}{*}{ Variable } & \multicolumn{2}{|l|}{ Contractility } & \multirow[b]{2}{*}{ p Value } \\
\hline & Impaired $(n=19)$ & Normal $(n=12)$ & \\
\hline Age (years) & $9.0(2.2)$ & $9.9(1.6)$ & 0.22 \\
\hline Sex F/M & $6 / 13$ & $1 / 11$ & 0.20 \\
\hline Body mass index $\left(\mathrm{kg} / \mathrm{m}^{2}\right)$ & $17.4(3.0)$ & $16.5(1.8)$ & 0.35 \\
\hline Age at operation (days) & $16.3(4.4)$ & $12.6(4.7)$ & $0.01^{*}$ \\
\hline Circulatory arrest time (min) & $6.6(5.2)$ & $4.0(1.3)$ & $0.045^{*}$ \\
\hline Cardiopulmonary bypass time (min) & $133.3(18.2)$ & $119.6(34.6)$ & $0.01^{*}$ \\
\hline Aortic cross clamp time (min) & $91.7(19.2)$ & $86.9(31.0)$ & 0.52 \\
\hline Unusual coronary artery pattern & $6(32 \%)$ & $0(0 \%)$ & 0.059 \\
\hline
\end{tabular}

Despite the encouraging early reports, the present study showed, to the contrary, subnormal LV contractility in a significant proportion of patients when followed up for nearly a decade after ASO. In fact, Vogel and colleagues ${ }^{18}$ have previously reported regional wall motion abnormalities and myocardial perfusion defects in seven of 21 children with TGA who were studied at a mean of 2.2 years after ASO. Bengel and colleagues ${ }^{19}$ reported impaired myocardial blood flow during adenosine induced vasodilatation and attenuation of coronary flow reserve measured by positron emission tomography in patients after one stage repair and, although their results may have been confounded by pulmonary artery banding before ASO in two thirds of their patients, their findings are a cause for concern. ${ }^{19}$ Furthermore, the same group of workers have reported high concentrations of glycogen phosphorylase isoenzyme $\mathrm{BB}$, a marker of myocardial ischaemia, at rest and after treadmill exercise in patients with stress induced defects on positron emission tomography. ${ }^{8}$ Importantly, significant coronary artery abnormalities were not found in any of these patients. Our findings hence corroborate those of the recent studies and reflect functional alteration of the left ventricle as a result of possible myocardial ischaemia, with further exaggeration under stress.

DSE studies have been used for early detection of cardiac dysfunction in children with cancer ${ }^{9}$ and thalassaemia. ${ }^{10}$ The optimal dose of dobutamine in children remains controversial. Nonetheless, frequent side effects have been reported to occur with an infusion rate of $\geqslant=10 \mu \mathrm{g} / \mathrm{kg} / \mathrm{min} .{ }^{9}$ In fact, De Wolf and colleagues ${ }^{20}$ showed that a dobutamine dose of $5 \mu \mathrm{g} / \mathrm{kg} / \mathrm{min}$ was tolerated and effective in inducing significant changes in systolic echocardiographic indexes in all of their patients, including children and young adults. ${ }^{20}$ To determine the presence of wall motion abnormalities, we analysed the wall motion at the maximum tolerated dose of dobutamine. The indications for termination of dobutamine in the present study were similar to those reported in the paediatric population. ${ }^{20}$

The underlying mechanisms for impairment of LV contractility, stress induced wall motion abnormalities, and myocardial perfusion defects remain speculative. The prevalence of coronary arterial stenosis and occlusion after ASO, as shown by aortography and selective coronary arteriography, is reported to range from 3-8\%. ${ }^{21}{ }^{22}$ It appears unlikely, however, that distortion of coronary artery anatomy explains our findings, as angiographic examination results were normal in our patients and evidence of involvement of a well defined coronary arterial territory was absent. Our findings, on the other hand, tend to support a more global impairment of LV function. The association between longer cardiopulmonary bypass and circulatory arrest times and impaired LV contractility may suggest that open heart surgery itself leads to LV dysfunction. Hayes and colleagues ${ }^{23}$ reported fixed myocardial perfusion defects in children after ASO and other types of open heart surgery and further speculated that microinfarction caused by embolism during open heart surgery is a potential explanation. However, the reversible perfusion defects detected in our patients suggest dynamic rather than static disturbance of myocardial blood flow.

Bengel and colleagues ${ }^{19}$ hypothesised that coronary vasoreactivity is altered as a result of endothelial dysfunction and sympathetic denervation relating to disconnection of coronary arteries during ASO. Their group's subsequent findings of impaired myocardial blood flow during adenosine induced vasodilatation and attenuation of coronary flow reserve in

Table 4 Exercise myocardial perfusion defects versus segmental wall motion abnormalities unmasked by dobutamine stress echocardiography

\begin{tabular}{cll}
\hline Patient & Exercise myocardial perfusion defects & Hypokinetic segments on dobutamine stress echocardiography \\
\hline 1 & Anterolateral & Apical ant, apical lat \\
2 & Ant, lat & Ant sept, apical lat, apical sept \\
4 & Ant, lat, inferolateral, basal inf & Ant sept, apical post, apical sept, apical lat \\
6 & Anterolateral, sept, inf, apical & Ant sept, mid post, apical post, apical inf, mid lat, mid inf, basal inf \\
7 & Anterolateral, anteroapical & Apical ant, mid ant, basal ant, apical inf, mid inf, basal inf \\
8 & Anterolateral & Apical ant, apical lat \\
10 & Ant, basal inferolateral & Mid ant, basal ant, mid inf, basal inf \\
12 & Ant & Mid ant sept, basal ant, basal ant sept \\
17 & Anteroseptal, inferolateral & Ant sept, apical inf, apical ant, mid ant, basal ant \\
18 & Anteroseptal, ant, inferoseptal & Apical sept, apical inf, mid inf, basal inf, apical ant sept, mid ant sept, basal ant sept \\
19 & Anterolateral, inf, inferoapical & Apical inf, apical ant, mid inf \\
20 & Ant, anterolateral & Mid ant, basal ant \\
22 & Anterolateral & Apical ant, apical lat \\
28 & Ant, inf & Apical ant, apical inf, mid inf, basal inf \\
29 & Anterolateral & Apical ant, mid ant, basal ant \\
30 & Ant, inf & Apical ant, apical inf, mid inf, basal inf \\
31 & Ant, inf & Ant sept, apical inf, mid inf, basal inf, apical ant \\
\hline \multirow{2}{*}{ Ant, anterior; inf, inferior; sept, septal; lat, lateral; mid, middle; post, posterior. }
\end{tabular}


patients after ASO lend further support to such alteration of coronary vasoreactivity. ${ }^{8}$ The use of the glucose tracer ${ }^{18} \mathrm{~F}$ deoxyglucose for identification of myocardial viability may even shed more light on the understanding of the pathogenetic mechanism. ${ }^{24}$ While our data suggest an association between myocardial perfusion defects and corresponding wall motion abnormalities, definite evidence of myocardial ischaemia is lacking. Additionally, causes other than ischaemia may result in perfusion abnormalities. Further studies to unveil the underlying mechanisms are clearly required.

Some limitations to this study deserve comment. Firstly, the stress-velocity index is not valid in the presence of wall motion abnormalities. Hence, LV contractility during dobutamine infusion cannot be accurately reflected by this index. Nonetheless, the value of DSE in unmasking wall motion abnormalities in patients is illustrated in the present study. Evaluating the accuracy of DSE in assessing wall motion abnormalities, however, would require appropriate statistical analysis with a $2 \times 2$ table of perfusion and wall motion defects assessed by exactly the same model of LV segmentation. Secondly, the amount of stress induced by dobutamine may not be exactly comparable with that induced during exercise due to heart rate limitation. However, the fact that similar segments with abnormal wall motion are identified separately by the two different modalities suggests that the amount of dobutamine stress is a reasonable choice. It is possible, however, that additional wall motion abnormalities and a higher wall motion score may result if a higher dobutamine dose, which would especially be more useful in young adults, were to be used. Furthermore, interpretation of the positive DSE and perfusion scan findings may be complicated by the lack of a definition of sensitivity and specificity of these investigations in detecting inducible myocardial ischaemia in children. Owing to ethical constraints, inclusion of a control group for interpretation of myocardial perfusion scan results in patients is difficult. The role of low dose DSE in predicting LV recovery after acute myocardial infarction ${ }^{25}$ and survival from revascularisation in adults with ischaemic cardiomyopathy ${ }^{26}$ has, nonetheless, been reported recently. Thirdly, aortic ET was not assessed simultaneously with $\mathrm{M}$ mode echocardiography but usually within a 30 second interval. Fourthly, there is an interval between selective coronary angiography and the timing of DSE and exercise myocardial perfusion scanning. However, the findings of DSE and perfusion scan, as alluded to earlier, do not support major stenosis or occlusion of main coronary artery branches.

In conclusion, a significant proportion of children, albeit asymptomatic, have LV contractile dysfunction, reversible myocardial perfusion defects, and mild stress induced wall motion abnormalities in the long term after ASO. The clinical correlate of these abnormalities, however, remains uncertain. Long term follow up of the outcomes of patients with TGA after ASO is hence indicated.

\section{ACKNOWLEDGEMENTS}

We thank Dr Elaine MC Chau for her technical support in wall motion analysis; nursing staff and secretaries of the Department of Paediatrics and Adolescent Medicine, Grantham Hospital, for their assistance; and our patients for their participation in this study. Funding of this research was partly provided by the Children's Heart Foundation.

\footnotetext{
Authors' affiliations

L Hui, A K T Chau, M P Leung, Y F Cheung, Division of Paediatric Cardiology, Grantham Hospital, The University of Hong Kong, Hong Kong, People's Republic of China

C S W Chiu, Division of Cardiothoracic Surgery, Grantham Hospital, The University of Hong Kong
}

\section{REFERENCES}

1 Di Donato RM, Wernovsky G, Walsh EP, et al. Results of the arterial switch operation for transposition of the great arteries with ventricular septal defect: surgical considerations and midterm follow-up data. Circulation 1989;80:1689-705.

2 Wernovsky G, Hougen TJ, Walsh EP, et al. Midterm results after the arterial switch operation for transposition of great arteries with intact ventricular septum: clinical, hemodynamic, echocardiographic, and electrophysiologic data. Circulation 1988;77:1333-44.

3 Lupinetti FM, Bove EL, Minich LL, et al. Intermediate-term survival and functional results after arterial repair for transposition of the great arteries. J Thorac Cardiovasc Surg 1992;103:421-7.

4 Colan SD, Trowitzsch E, Wernovsky G, et al. Myocardial performance after arterial switch operation for transposition of the great arteries with intact ventricular septum. Circulation 1988;78:132-41.

5 Hovels-Gurich HH, Seghaye MC, Dabritz S, et al. Cardiological and general health status in preschool- and school-age children after neonatal arterial switch operation. Eur J Cardiothorac Surg 1997;12:593-601.

6 Colan SD, Boutin C, Castaneda AR, et al. Status of the left ventricle after arterial switch operation for transposition of the great arteries: hemodynamic and echocardiographic evaluation. J Thorac Cardiovasc Surg 1995; 109:311-21.

7 Crepaz R, Secchieri S, Svaluto G, et al. Echocardiographic evaluation of systolic and diastolic left ventricular function following arterial switch operation in neonatal period for transposition of the great arteries. G Ital Cardiol 1997;27:224-30.

8 Hauser M, Bengel FM, Kuhn A, et al. Myocardial blood flow and flow reserve after coronary reimplantation in patients after arterial switch and Ross operation. Circulation 2001;103:1875-80.

9 Klewer SE, Goldberg SJ, Donnerstein RL, et al. Dobutamine stress echocardiography: a sensitive indicator of diminished myocardial function in asymptomatic doxorubicin-treated long-term survivors of children cancer. J Am Coll Cardiol 1992; 19:394-401.

10 Hui L, Leung MP, Ha SY, et al. Early detection of left ventricular dysfunction in patients with $\beta$ thalassaemia major by dobutamine stress echocardiography. Heart 2003:89:669-70.

11 Elhendy A, Zoet-Nugeteren S, Cornel JH, et al. Functional assessment of ALCAPA syndrome by dobutamine stress thallium-201 SPECT and echocardiography. J Nucl Med 1996;37:748-51.

12 Noto N, Ayusawa M, Larasawa K, et al. Dobutamine stress echocardiography for detection of coronary artery stenosis in children with Kawasaki disease. J Am Coll Cardiol 1996;27:1251-6.

13 Gittenberger-de Groot AC, Sauer U, Oppenheimer-Dekker A, et al. Coronary arterial anatomy in transposition of the great arteries: a morphologic study. Pediatr Cardiol 1983;4(suppl 1):15-24.

14 Sahn DJ, DeMaria A, Kisslo J, et al. Recommendations regarding quantitation in M-mode echocardiography: results of a survey of echocardiographic measurements. Circulation 1978;58:1072-83.

15 Sandor GGS, Popov R, De Souza E, et al. Rate-corrected mean velocity of fiber shortening-stress at peak systole as a load-independent measure of contractility. Am J Cardiol 1992;69:403-7.

16 Colan SD, Kenneth MB, Neumann A. Left ventricular end-systolic wall stress velocity of fiber shortening relationship: a load-independent index of myocardial contractility. J Am Coll Cardiol 1984;4:715-24.

17 Schiller NB, Shah PM, Crawford M, et al. Recommendations for quantitation of the left ventricle by two-dimensional echocardiography. J Am Soc Echocardiogr 1989;2:358-67.

18 Vogel M, Smallhorn JF, Trusler GA, et al. Echocardiographic analysis of regional left ventricular wall motion in children after the arterial switch operation for complete transposition of the great arteries. J Am Coll Cardiol 1990; 15:1417-23.

19 Bengel FM, Hauser M, Duvernoy CS, et al. Myocardial blood flow and coronary flow reserve late after anatomical correction of transposition of the great arteries. J Am Coll Cardiol 1998;32:1955-61.

20 De Wolf $D$, Suys B, Verhaaren $H$, et al. Low-dose dobutamine stress echocardiography in children and young adults. Am J Cardiol 1998;81:895-901.

21 Bonnet D, Bonhoeffer J, Piechaud J-F, et al. Long-term fate of the coronary arteries after the arterial switch operation in newborns with transposition of the great arteries. Heart 1996;76:274-9.

22 Tanel RE, Wernovsky G, Landzberg MJ, et al. Coronary artery abnormalities detected at cardiac catheterization following the arterial switch operation for transposition of treat arteries. Am J Cardiol 1995;76:153-7.

23 Hayes AM, Baker EJ, Kakadeker A, et al. Influence of anatomic correction for transposition of great arteries on myocardial perfusion: radionuclide imaging with technetium-99m 2-methoxy isobutyl isonitrile. J Am Coll Cardiol 1994;24:769-77

24 Hernandez-Pampaloni M, Allada V, Fishbein MC, et al. Myocardial perfusion and viability by positron emission tomography in infants and children with coronary abnormalities. J Am Coll Cardiol 2003;41:618-26.

25 Hillis GS, Mulvagh SL, Pellikka PA, et al. Comparison of intravenous myocardial contrast echocardiography and low-dose dobutamine echocardiography for predicting left ventricular functional recovery following acute myocardial infarction. Am J Cardiol 2003;92:504-8.

26 Sawada SG, Lewis SJ, Foltz J, et al. Usefulness of rest and low-dose dobutamine wall motion score in predicting survival and benefit from revascularization in patients with ischemic cardiomyopathy. Am J Cardiol 2002;89:811-6. 